\title{
QUALIDADE DE VIDA DE PESSOAS COM LESÃO MEDULAR TRAUMÁTICA*
}

\author{
Lionela da Silva Corrêa ${ }^{1}$, David Lopes Neto ${ }^{2}$, Eliana Ofélia Llapa-Rodriguez ${ }^{3}$
}

${ }^{1}$ Educadora Física. Mestre em Ciência da Saúde. Docente da Universidade Federal do Amazonas. Manaus, AM, Brasil. Enfermeiro. Doutor em Enfermagem. Docente da Universidade Federal do Amazonas, Manaus, AM, Brasil.

${ }^{3}$ Enfermeira. Doutora em Enfermagem. Docente da Universidade Federal de Sergipe. Aracaju, SE, Brasil.

RESUMO: Estudo descritivo, quantitativo, cujo objetivo era avaliar a qualidade de vida de pessoas com lesão medular traumática atendidas em um hospital universitário de Manaus, estado do Amazonas, entre agosto a outubro de 2011. Participaram da pesquisa 30 paraplégicos, com lesão medular traumática. Para mensurar a qualidade de vida foi utilizado o questionário World Health Organization Quality of Life-Bref, dividido em quatro domínios: físico, psicológico, social e meio ambiente. Os resultados mostram predominância do gênero masculino, com idade entre 31 e 45 anos, com cinco a oito anos de lesão. Dos 30 entrevistados, 20 e 36,7\% avaliaram a qualidade de vida como muito boa e boa, respectivamente. Entre os domínios destacaram-se o psicológico, o social e o meio ambiente, com avaliações de qualidade de vida boa (56,7\%), média (43,3\%) e média (53,3\%). Conclui-se que os lesados medulares se percebem com qualidade de vida satisfatória nos conjunto dos domínios psicológico e social.

DESCRITORES: Qualidade de vida; Traumatismos da medula espinal; Satisfação pessoal.

\section{QUALITY OF LIFE OF PERSONS WITH TRAUMATIC SPINAL CORD INJURIES}

ABSTRACT: This descriptive quantitative study aimed to evaluate the quality of life of persons with traumatic spinal cord injuries attended in a teaching hospital in Manaus, in the Brazilian state of Amazonas, between August and October 2011. A total of 30 paraplegics with traumatic spinal cord injuries participated in the study. The World Health Organization Quality of Life-Bref questionnaire was used for measuring quality of life. This is divided into four domains: physical health, psychological health, social relationships and environment. The results showed a predominance of the male gender, aged between 31 and 45 years old, who had lived with the injury for five to eight years. Of the 30 interviewees, $20 \%$ and $36.7 \%$ assessed quality of life as 'very good' and 'good' respectively. Among the domains, the psychological, social and environmental stood out, with evaluations of quality of life as good (56.7\%), average (43.3\%) and average (53.3\%). It is concluded that persons with spinal cord injuries perceive themselves as having a satisfactory quality of life in the sets of the psychological and social domains.

DESCRIPTORS: Quality of life; Spinal cord injuries; Personal satisfaction.

\section{CUALIDAD DE VIDA DE PERSONAS CON LESIÓN MEDULAR TRAUMÁTICA}

RESUMEN: Estudio descriptivo, cuantitativo, cuyo objetivo fue evaluar la cualidad de vida de personas con lesión medular traumática atendidas en un hospital universitario de Manaus, estado de Amazonas, entre agosto y octubre de 2011. Participaron de la investigación 30 parapléjicos, con lesión medular traumática. Para mensurar la cualidad de vida, fue utilizado el cuestionario World Health Organization Quality of Life-Bref, organizado en cuatro dominios: físico, psicológico, social y de medio ambiente. Los resultados muestran predominancia del género masculino, con edad entre 31 y 45 años, con cinco a ocho años de lesión. De los 30 entrevistados, 20 y $36,7 \%$ evaluaron la cualidad de vida como muy buena y buena, respectivamente. Entre los dominios se destacaron el psicológico, el social y el del medio ambiente, con evaluaciones de cualidad de vida buena $(56,7 \%)$, media $(43,3 \%)$ y media $(53,3 \%)$. Se concluye que las personas con lesión medular se perciben con cualidad de vida satisfactoria en los conjuntos de dominios psicológico y social.

DESCRIPTORES: Cualidad de vida; Traumatismos de la médula espinal; Satisfacción personal.

*Artigo extraído da dissertação intitulada: "Estudo da qualidade de vida de pessoa com lesão medular." Universidade Federal do Amazonas, 2011.

Autor Correspondente:

David Lopes Neto

Universidade Federal do Amazonas

R. Doutor Thomas, 215, ap 504-B - 69.053-035 - Manaus, AM, Brasil

E-mail: davidnetto@uol.com.br
Recebido: $17 / 05 / 2015$ Finalizado: 20/08/2015 


\section{INTRODUÇÃO}

A lesão medular traumática é um problema catastrófico. Em 2011, a taxa de incidência mundial foi estimada em 179.312 casos novos por ano. Os dados epidemiológicos mostram a magnitude do problema em número de casos por ano (por um milhão de pessoas): América do Norte (40), Europa Ocidental (16), Austrália (15), Ásia-Central (25), Ásia-Sul (21), Caribe (19), América Latina e Andina (19), América Latina e Central (24) ${ }^{(1)}$.

O traumatismo da medula espinhal compromete o funcionamento das funções motora, sensitiva, visceral, sexual e trófica ${ }^{(2)}$, estando relacionado as causas decorrentes de violência urbana, acidentes de trânsito e agressão por arma de fogo ${ }^{(3-6)}$, acometendo mais os homens, solteiros, residentes em áreas urbanas com idade entre 18 e 35 anos $^{(6-8)}$.

Os casos de lesão medular traumática vêm aumentando e se tornando um problema de saúde pública devido ao número crescente de ocorrências de violência urbana. No Brasil, não existem dados atualizados ou consolidados; no entanto, cerca de 7.000 casos de pessoas diagnosticadas com lesão medular traumática são relatados por ano ${ }^{(9)}$.

A inclusão de pessoas com lesão medular em programas de reabilitação significa, além da possibilidade de regressão do déficit neurológico, a redução do grau de dependência, a melhora da sociabilidade e a diminuição de comorbidades clínicas $^{(5)}$, haja visto que sequelas decorrentes de traumas podem interferir na qualidade de vida das pessoas ${ }^{(3)}$.

Qualidade de vida é um conceito multidimensional que aborda saúde, estilo de vida, funcionamento social e condições de saúde, e se expressa na forma como a pessoa se percebe e se posiciona na vida frente ao contexto cultural e ao sistema de valores nos quais vive e em relação aos seus objetivos, expectativas, padrões e preocupações ${ }^{(10)}$.

O objetivo deste estudo foi avaliar a qualidade de vida de pessoas com lesão medular traumática atendidas em um hospital universitário de Manaus-AM.

\section{MÉTODOS}

Estudo descritivo, de abordagem quantitativa, realizado no período de agosto a outubro de 2011, com 30 pacientes com diagnóstico de lesão medular traumática internados no Hospital Universitário Getúlio Vargas (HUGV) - ManausAM. Utilizou-se como critérios de inclusão pessoas internadas no período de 2000 a 2010, com diagnóstico de lesão medular traumática, com idade igual ou superior a 18 anos e com endereço definido na cidade de Manaus, estado do Amazonas.

A amostra inicial de pacientes acometidos por lesão medular foi de 381, dos quais, 194 tiveram seus cadastros completos no prontuário. Após triagem, 45 sujeitos foram considerados aptos a participar da pesquisa. No entanto, 15 não aceitaram participar do estudo quando não assinaram o termo de consentimento livre e esclarecido. O $\mathrm{n}$ final da pesquisa foi de 30 sujeitos.

$\mathrm{O}$ instrumento utilizado para mensurar a qualidade de vida foi a versão brasileira ${ }^{(11)}$ da World Health Organization Quality of Life-Bref (WHOQOL-Bref), com 26 perguntas, das quais, 24 foram distribuídas em quatro domínios: físico, psicológico, relações sociais e meio-ambiente. Os domínios são representados por facetas e suas questões em escala de respostas do tipo Likert de intensidade (nada-extremamente), capacidade (nada-completamente), frequência (nunca-sempre) e de avaliação (muito insatisfeitomuito satisfeito; muito ruim-muito bom). Nas respostas, um corresponde à negatividade $\mathrm{e}$ cinco à positividade, sendo que quanto menores os percentis de avaliação, menores ou inferiores os níveis na qualidade de vida. Além dos quatro domínios, trabalhou-se, no estudo, com a questão relativa à percepção da qualidade de vida: "Quão satisfeito(a) você está com a sua saúde?", da qual as respostas variaram entre muito satisfeito a muito insatisfeito ${ }^{(2)}$.

Para verificar a correlação entre qualidade de vida e as variáveis (idade, independência funcional, gênero, renda, tempo de lesão e sequela), foi utilizado o coeficiente de correlação de Spearman, com o nível de significância de 5\% $(\mathrm{p}=<0,05)$.

A análise dos dados foi realizada por meio do emprego do programa Statistical Package For Social Sciences (SPSS), versão 16.0. As análises estatísticas incluíram análises descritivas de medida de tendência central (média), medida de posição (valor mínimo e máximo) e medida de dispersão (desvio padrão).

Este artigo foi extraído da dissertação de mestrado intitulada: Estudo da qualidade de vida 
de pessoa com lesão medular, 2011. O projeto foi aprovado pelo Comitê de Ética em Pesquisa da Universidade Federal do Amazonas sob CAAE 0278.0.115.000-11.

\section{RESULTADOS}

Dos 30 sujeitos com lesão medular traumática, predominou o gênero masculino (83,3\%); com idades entre 31 e 45 anos (56,7\%); com tempo de lesão de cinco a oito anos (40\%), com sequela de paraplegia $(50 \%)$, com renda de até dois salários mínimos $(60 \%)$ e com dependência completa/ modificada (53,3\%). Quanto às causas da lesão medular, a principal foi por queda $(47 \%)$, seguida por acidentes de trânsito $(20 \%)$ e por arma de fogo $(13 \%)$, o restante das causas somaram $20 \%$.

Dos 30 entrevistados, $20 \%$ avaliou a qualidade de vida geral como muito boa (6), 36,7\% como boa (11) e 3,3\% como ruim (1). Houve $40 \%$ dos participantes que manifestaram ter uma qualidade de vida média.

Quando analisados os domínios, no domínio físico, os sujeitos avaliaram a qualidade de vida como ruim $(30 \%)$, média $(30 \%)$, boa $(30 \%)$ e muito boa $(10 \%)$. Destacando as maiores médias nas facetas: energia e fadiga $(3,6)$, disposição diante da dor $(3,5)$, sono e repouso $(3,3)$, atividades de vida cotidiana $(3,2)$ e mobilidade $(3,1)$. Contrariamente, as facetas nas quais os sujeitos apresentaram maior insatisfação foram: dependência de medicação ou tratamentos e capacidade de trabalho, com uma média de 2,9 para cada faceta.

Em relação ao domínio psicológico, os sujeitos avaliaram a qualidade de vida como boa (56,7\%), $20 \%$ avaliaram como média, $13,3 \%$ como muito boa e $10 \%$ como ruim. Em geral, para este domínio, os participantes mostraram médias acima de 3,2 em todas as facetas (sentimentos positivos; crenças pessoais; concentração; aceitação de aparência física; autoestima e ausência de sentimentos negativos).

No domínio social, composto pelas facetas relações pessoais, atividade sexual e apoio social, os sujeitos $(43,3 \%)$ avaliaram a qualidade de vida como média, ou seja, não se consideraram satisfeitos, contudo, não mencionaram insatisfação. Apesar desse resultado, verificou-se que as maiores médias estão nas facetas relações pessoais $(3,5)$ e apoio social $(3,3)$ e as menores médias na faceta vida sexual $(2,8)$.

Quantoaodomíniomeioambiente, relacionado à segurança física e proteção, ambiente no lar, recursos financeiros, disponibilidades e qualidade de cuidados de saúde e sociais, oportunidades de adquirir novas informações e habilidades, oportunidades de recreação e lazer e, ambiente físico e transporte, na avaliação dos sujeitos, a qualidade de vida foi considerada como média para $53,3 \%$ e $30 \%$ a avaliaram como ruim. Quanto aos itens do domínio meio ambiente, observaramse as menores médias nas variáveis recursos financeiros $(2,1)$; atividade de lazer $(2,1)$; acesso aos serviços de saúde $(2,2)$ e meio de transporte $(1,9)$. Por outro lado, as maiores médias foram para as questões referentes à oportunidade de novas informações $(3,7)$; segurança física $(3,2)$; ambiente físico $(3,2)$ e condições de moradia $(3,1)$.

Quando verificada a satisfação do sujeito em relação a sua saúde, $40 \%$ dos entrevistados responderam não estarem satisfeitos nem insatisfeitos com a saúde, mostrando uma média de 3,1 nas respostas.

Das variáveis sociais estudadas (idade, independência funcional, gênero, renda, tempo de lesão e sequela), todas foram significativas; em destaque, a idade por ser considerada uma variável muito significativa, quando relacionada com a qualidade de vida (Tabela 1 ).

Tabela 1 - Coeficiente de correlação entre qualidade de vida e as variáveis. Manaus, AM, Brasil, 2011

\section{Correlação entre qualidade de vida e variáveis}

\begin{tabular}{llc}
\hline Idade & Coeficiente de correlação & $-0,461$ \\
\cline { 2 - 3 } & Significância & 0,01 \\
\hline Independência & Coeficiente de Correlação & 0,008 \\
\cline { 2 - 3 } Funcional & Significância & 0,968 \\
\hline Gênero & Coeficiente de Correlação & 0,047 \\
\cline { 2 - 3 } & Significância & 0,807 \\
\hline Renda (em & Coeficiente de Correlação & $-0,069$ \\
\cline { 2 - 3 } $\begin{array}{l}\text { Salários } \\
\text { Mínimos) }\end{array}$ & Significância & 0,718 \\
\hline Tempo de & Coeficiente de correlação & $-0,311$ \\
\cline { 2 - 3 } $\begin{array}{l}\text { Lesão (em } \\
\text { anos) }\end{array}$ & Significância & 0,094 \\
\hline Sequela & Coeficiente de Correlação & 0,208 \\
\cline { 2 - 3 } & Significância & 0,269
\end{tabular}




\section{DISCUSSÃO}

A qualidade de vida é uma construção multifacetada que engloba capacidades comportamentais e cognitivas do indivíduo, bem-estar emocional e habilidades que exigem o desempenho de papéis domésticos, profissionais e sociais, sendo, portanto, um conceito dinâmico que incorpora não apenas domínios físicos, psicológicos e sociais, mas as percepções e os valores individuais ${ }^{(12)}$.

Nesse contexto, em relação aos sujeitos com lesão medular traumática, as sequelas decorrentes do trauma e as dificuldades sociais podem influenciar negativamente nos níveis de qualidade de vida, acarretando comprometimentos dos aspectos sociais e físicos ${ }^{(13-14)}$. A literatura menciona que o lesado medular pode vivenciar, no seu dia a dia, problemas físicos e orgânicos, como úlceras de pressão, infecção urinária, espasticidade e deformidades, condições que podem, na maioria dos casos, ocasionar dificuldade ou incapacidade para realização de movimentos, situações que são percebidas de formas negativas por este tipo de paciente, afetando, inclusive, sua saúde mental ${ }^{(15-16)}$. Assim, infere-se que esses sejam alguns dos motivos de insatisfações dos sujeitos quanto às facetas capacidade de trabalhar e dependência de medicação ou tratamentos.

Em relação ao domínio psicológico, a maioria dos sujeitos avaliou a qualidade de vida como positiva, resultado similar foi encontrado em outro estudo ${ }^{(3)}$. Em geral, para este domínio, os participantes mostraram-se satisfeitos em todas as facetas (sentimentos positivos; pensamento, aprendizagem, memória e concentração; autoestima; aparência e imagem corporal; ausência de sentimentos negativos; espiritualidade, religião e crenças pessoais).

No domínio social, a demonstração de satisfação (nas facetas relações pessoais e apoio social) foi similar ao estudo sobre qualidade de vida, realizado com chineses diagnosticados com lesão medular, o qual mostrou que a inclusão social e profissional são aspectos relevantes para a qualidade de $\operatorname{vida}^{(17)}$. Outras pesquisas ${ }^{(15,18)}$ mostraram que há dificuldades quanto ao domínio social, ao mencionarem a existência de insatisfação com a vida sexual, indicando entre as causas: ausência de relações sexuais; disfunção sexual como a não ejaculação e a falta de prazer ${ }^{(15)}$. Outros estudos ${ }^{(19-20)}$ apontaram resultados semelhantes aos da presente pesquisa.

Quanto ao domínio meio ambiente, a avaliação de qualidade de vida como média, corroborou com os resultados encontrados em estudo sobre pacientes com lesão medular ${ }^{(3)}$. A esse respeito, foi percebido que as facetas avaliadas como positivas foram as atividades que o indivíduo realizava em casa, a exemplo de ligar a televisão, acessar a internet, ouvir o rádio ou ler um jornal. Por outro lado, foram avaliadas como negativas as facetas cujas atividades aconteciam fora da residência, tais como: o acesso ao serviço de saúde, a prática de atividades de lazer e o transporte, por estas dependerem de outros fatores para serem executadas de forma satisfatória.

Por outro lado, a acessibilidade é fundamental para que uma pessoa em cadeira de roda ou com dificuldade na locomoção possa transitar de forma autônomaerealizaratividades relacionadasàsaúde ou ao lazer. A inacessibilidade tem implicações sobre a qualidade de vida, uma vez que restringe as oportunidades prazerosas e importantes para a sobrevivência. Pesquisadores(15) identificaram algumas dificuldades dos lesados medulares para o acesso aos serviços de saúde, tais como: dificuldades de transporte, falta de acessibilidade aos locais, existência de fila para $\mathrm{o}$ atendimento e fragilidades no atendimento especializado. Portanto, modificações em ambientes são necessárias para torná-los mais amplos e acessíveis, a exemplo do acesso a prédios públicos e transporte coletivo, para que pessoas em situação de vulnerabilidade de física e motora tenham seus direitos garantidos e suas necessidades atendidas ${ }^{(21-22)}$.

$\mathrm{Na}$ avaliação do domínio meio ambiente, observou-se que os sujeitos se mostraram satisfeitos em relação à segurança, ao ambiente físico (clima, barulho, poluição, atrativos) e à disponibilidade de informações; mas se mostraram insatisfeitos quanto aos recursos financeiros, oportunidade de praticar atividades de lazer, acesso aos serviços de saúde e meio de transporte, tendo em vista que estas são situações em que o lesado medular depende de outros fatores para realizar sua funcionalidade de forma satisfatória. Logo, a relação com o meio ambiente é outro fator que pode afetar a qualidade de vida do lesado medular.

Os resultados da presente pesquisa evidenciaram que as variáveis independência funcional, gênero, renda, tempo de lesão e sequela da lesão não apresentaram relação direta com qualidade de vida dos sujeitos com lesão medular, fortalecendo o conceito de que qualidade de vida é subjetiva e, por conseguinte, 
individual.

A percepção é um processo mental do mundo percebido. Nessa perspectiva, de abstração da realidade vivenciada, analisando um sujeito com lesão medular que não apresenta independência funcional, mas não a percebe como algo negativo e que afeta sua qualidade de vida e outro sujeito na mesma condição, mas que tem a percepção negativa da independência funcional para a sua vida, pode-se afirmar que a qualidade de vida não está relacionada com a condição de dependência funcional, mas com a forma como o meio é percebido. Dessa forma, a percepção de qualidade de vida está mais relacionada com as fases de superação do trauma do que com as condições de funcionalidade, gênero, renda ou tempo de lesão. Por este prisma, a qualidade de vida pode ser percebida de diferentes formas e por diferentes pessoas em cenários idênticos, em um mesmo momento, sendo uma resposta advinda da subjetividade humana.

A percepção positiva ou negativa de qualidade de vida dos sujeitos está relacionada à idade, haja vista que pacientes mais jovens apresentam melhor qualidade de vida e, por conseguinte, melhor inserção social(7), condição que está atrelada à redução da reserva fisiológica, à diminuição da vitalidade e ao envelhecimento do corpo $^{(14)}$.

Ao comparar o nível de qualidade de vida dos lesados medulares deste estudo com os de outras pesquisas ${ }^{(23-25)}$, evidenciou-se que em relação ao domínio físico e meio ambiente, os resultados desta pesquisa foram mais negativos. No domínio psicológico, os resultados foram melhores em relação às vítimas de trauma, aos pacientes com transtorno de pânico e aos pacientes com diabetes mellitus e hipertensão arterial associados. No domínio relação social, o resultado foi superior para os pacientes com câncer colorretal, isso ocorre devido ao lesado medular vivenciar problemas físicos e orgânicos, como: presença de úlceras de pressão, infecção urinária, espasticidade e deformidades ${ }^{(15)} \mathrm{e}$, além disso, apresentar dificuldade ou incapacidade para a realização de movimentos físicos, o que poderá ser percebido de forma negativa para possíveis potenciais, alternativas e obter satisfação de suas necessidades e anseios ${ }^{(26)}$. Ressalta-se que a qualidade de vida no lesado medular tem relação com mobilidade, integração social e ocupação, as quais são papéis sociais significativos.

\section{CONCLUSÃO}

Os lesados medulares se perceberam com boa qualidade de vida e saúde satisfatória. Na avaliação do conjunto dos domínios físico, psicológico, relações sociais e meio ambiente, os resultados evidenciaram que os lesados medulares avaliaram como satisfatórios os domínios psicológico e social, demonstrando insatisfação com as condições ambientais (domínio meio ambiente).

O domínio meio ambiente se apresentou como barreira para satisfação plena da qualidade de vida. Diferentemente, o domínio físico não foi definido como satisfatório nem insatisfatório pelos participantes. Os resultados deste estudo sugerem que a idade influencia diretamente na qualidade de vida do lesado medular.

Conclui-se que embora o número de entrevistados seja um limitador desta pesquisa, espera-se que seus resultados colaborem com políticas públicas de saúde voltadas para a criação de programas que reintegrem os sujeitos com lesão medular à condição social e de trabalho, permitindo a sua independência social e profissional.

Pesquisas futuras são necessárias para avaliarem melhor a forma como as pessoas com lesão medular traumática vivenciam as mudanças que ocorrem nas suas vidas ao longo do tempo.

\section{REFERÊNCIAS}

1. Lee BB, Cripps RR, Fitzharris M, Wing PC. The global map for traumatic spinal cord injury epidemiology: update 2011, global incidence rate. Spinal Cord. 2014; 52(2):110-16.

2. Assis GM, Faro ACM. Clean intermittent selfcatherization in spinal cord injury. Rev. esc. Enferm. USP. 2011; 45(1):282-6.

3. Bampi LNS, Guilhem D, Lima DD. Quality of live in people with traumatic spinal cord injury: a study with WHOQOL-BREF. Rev. bras. epidemiol. 2008; 11(1):6777.

4. Vall J, Costa CMC, Pereira LF, Friesen TT. Application of international classification of functioning, disability and health (ICF) in individuals with spinal cord injury. Arq Neuropsiquiatr. 2011; 69(3):513-8.

5. Ninomyia AF, Jesus CLM, Auletta LL, Rimkus CM, Ferreira DM, Zoppi Filho A, et al. Shoulders of patients with spinal cord injuries submitted to rehabilitation program - a clinical and ultrasound-based assessment. Acta Ortop Bras. 2007; 15(2):109-13. 
6. Coura AS, França ISX, Enders BC, Barbosa ML, Souza JRS. Functional disability of adult individuals with spinal cord injury and its association with socio-demographic characteristics. Rev. Latino-Am. Enfermagem 2012; 20(1):84-92.

7. Brito LMO, Chein MBC, Marinho SC, Duarte TB. Epidemiological evaluation of victims of spinal cord injury. Rev. Col. Bras. Cir. 2011; 38(5):304-9.

8. Vall J, Braga VAB, Almeida PC. Study of the quality of life in people with traumatic spinal cord injury. Arq Neuropsiquiatr. 2006; 64(2b):451-55.

9. França ISX, Enders BC, Coura AS, Cruz GKP, Aragão JS, Oliveira DRC. Lifestyle and health conditions of adults with spinal cord injury. Invest Educ Enferm. 2014; 3(2):244-51.

10. Pereira EF, Teixeira CS, Santos A. Qualidade de vida: abordagens, conceitos e avaliação. Rev. bras. Educ. Fís. Esporte, 2012; 26(2):241-50.

11. Fleck MPA, Louzada $S$, Xavier $M$, Chachamovich E, Vieira G, Santos L, et al. Aplicação da versão em português do instrumento abreviado de avaliação da qualidade de vida "WHOQOL-brief". Rev. Saúde Pública. 2000; 34(2):178-83.

12. Chappell P, Wirz S. Quality of life following spinal cord injury for 20-40 year old males living in Sri Lanka. Asia Pacific Disability Rehabilitation Journal. 2003; 14(2):162-78.

13. Brunozi AE, Silva AC, Gonçalves LF, Veronezi RJB. Quality of life in the traumatic spinal cord injury. Rev Neurocienc. 2011; 19(1):139-44.

14. Middleton J, Tran Y, Craig A. Relationship between quality of life and self-efficacy in persons with spinal cord injuries. Arch Phys Med Rehabil. 2007; 88(1):164348.

15. Venturini DA, Decesaro MN, Marcon SS. Conhecendo a história e as condições de vida de indivíduos com lesão medular. Rev. Gaúcha Enferm. 2006; 27(2):219-29.

16. Ghazwin MY, Chaibakhsh S, Latifi S, Tavakoli AH, Koushki Arch Neurosci. Quality of life in Iranian men with spinal cord injury in comparison with general population. 2015; 2(2):e21529.

17.Tasiemski T, Nielsen S, Wilski M. Quality of life in people with spinal cord injury - earthquake survivors from Sichuan Province in china. Asia Pacific Disability Rehabilitation Journal. 2010; 21(2):28-36.

18. Kawanishi C, Greguol M. Avaliação da autonomia funcional de adultos com lesão medular. Rev Ter Ocup Univ São Paulo. 2014; 25(2):159-66.
19. Oliveira PP, Sandrin CC, Batista PH, Marinheiro LPF, Wender MCO, Roisenberg F. Relação entre qualidade de vida e fratura vertebral em mulheres idosas residentes no Sul do Brasil. Rev. Bras. Ginecol. Obstet. 2011; 33(5):231-7.

20. França ISX, Coura AS, França EG, Basílio NNV, Souto RQ. Quality of life of adults with spinal cord injury: a study using the WHOQOL-bref. Rev. esc. Enferm. USP. 2011; 45(6):1364-71.

21.Murta SG, Guimarães SS. Enfrentamento à lesão medular traumática. Estud. psicol. (Natal). 2007; 1(12):57-63.

22. França ISX, Baptista RS, Abrão FMS, Coura AS, França EG, Pagliuca LMF. The no-care of patients with spinal cord injuries in primary care: bioethical challenges for health policies. Rev. bras. enferm. 2012; 65(2):236-43.

23. Melo Neto VL, Valença AM, Nascimento I, Lopes FL. Quality of life assessment by WHOQOL-BREF in panic disorder patients during treatment. Rev. psiquiatr. clín. 2008; 35(2):49-54.

24. Santos CMS, França Junior I, Lopes F. Quality of life of people living with HIV/AIDS in São Paulo, Brazil. Rev. Saúde Pública. 2007; 41(Suppl 2):64-71.

25. Miranzi SSC, Ferreira FS, Iwamoto HH, Pereira GA, Miranzi MAS. Quality of life for diabetic and hypertensions individual accompanied by the family health team. Texto context - enferm. 2008; 17(4):672-9.

26. Fechio MB, Pacheco KMB, Kaihami HN, Alves VLR. The repercussions of a spinal cord injury over the individual's identity. Acta Fisiátr. 2009; 16(1):38-42. 